\title{
Carrier recombination parameters in diamond after surface boron implantation and annealing
}

Cite as: J. Appl. Phys. 127, 245707 (2020); https://doi.org/10.1063/5.0004881

Submitted: 19 February 2020 . Accepted: 10 June 2020 . Published Online: 29 June 2020

P. Grivickas (D), P. Ščajev (D) N. Kazuchits (D), A. Mazanik (D), O. Korolik (D), L. F. Voss (D), A. M. Conway, D. L. Hall, M. Bora, L. Subačius, V. Bikbajevas, and V. Grivickas (D)
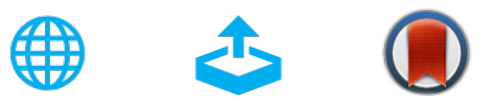

View Online

Export Citation

\section{ARTICLES YOU MAY BE INTERESTED IN}

Low-loss superconducting titanium nitride grown using plasma-assisted molecular beam epitaxy

Journal of Applied Physics 127, 235302 (2020); https://doi.org/10.1063/5.0008010

The piezoresistive mobility modeling for cubic and hexagonal silicon carbide crystals Journal of Applied Physics 127, 245113 (2020); https://doi.org/10.1063/5.0006830

Shock compression of paraffin-poly-methylmethacrylate (PMMA) mixture

Journal of Applied Physics 127, 245902 (2020); https://doi.org/10.1063/5.0013020

\section{Lock-in Amplifiers up to $600 \mathrm{MHz}$}
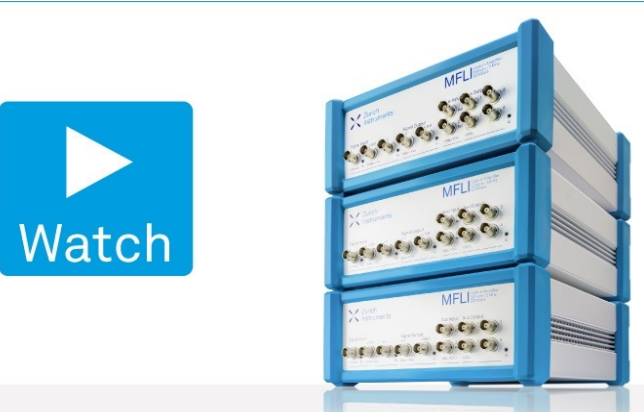


\title{
Carrier recombination parameters in diamond after surface boron implantation and annealing
}

Cite as: J. Appl. Phys. 127, 245707 (2020); doi: 10.1063/5.0004881

Submitted: 19 February 2020 . Accepted: 10 June 2020 .

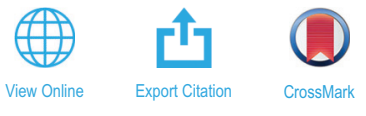

Published Online: 29 June 2020

P. Grivickas, ${ }^{1, a)}$ (D) P. Ščajev, ${ }^{2}$ (D) N. Kazuchits, ${ }^{3}$ (D) A. Mazanik, ${ }^{3}$ (D) O. Korolik, ${ }^{3}$ (D) L. F. Voss, ${ }^{1}$ (D) A. M. Conway,

D. L. Hall, ${ }^{7}$ M. Bora, ${ }^{1}$ L. Subačius, ${ }^{4}$ V. Bikbajevas, ${ }^{2}$ and V. Grivickas ${ }^{2}$ (D)

\author{
AFFILIATIONS \\ ${ }^{7}$ Lawrence Livermore National Laboratory, 7000 East Ave., Livermore, California 94551, USA \\ ${ }^{2}$ Institute of Photonics and Nanotechnology, Vilnius University, Sauletekio av. 3, Vilnius 10257, Lithuania \\ ${ }^{3}$ Department of Physics, Belarusian State University, Nezalezhnastsi av. 4, Minsk 220030, Belarus \\ ${ }^{4}$ Center for Physical Sciences and Technology, Sauletekio av. 3, Vilnius 10257, Lithuania
}

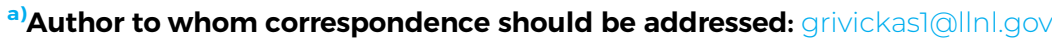

\begin{abstract}
An optical pump-probe technique was used to detect spatial distribution of carrier lifetimes across the thickness of a high-quality diamond device structure. Two samples with as-received and boron implanted surfaces were compared to assess the role of implantation and related processing on carrier recombination mechanisms. It was found that the two implanted surfaces show very different behaviors despite undergoing the same treatment. At one of the surfaces, carrier lifetimes remained relatively unchanged, indicating surface recombination rates in the $10^{2}-10^{3} \mathrm{~cm} / \mathrm{s}$ range. At the other surface, carrier lifetimes were almost a magnitude lower and correlated with the locally detected nitrogen vacancy defect that was attributed to the elevated concentration of residual nitrogen.
\end{abstract}

Published under license by AIP Publishing. https://doi.org/10.1063/5.0004881

\section{INTRODUCTION}

In diamond, boron (B) is the shallowest known acceptor with an activation energy of $370 \mathrm{meV} .^{1-3}$ The activation energy decreases with $\mathrm{B}$ concentration ${ }^{2,4}$ leading into Mott transition for a metallic state at concentrations above $2 \times 10^{20} \mathrm{~cm}^{-3}$. Boron implantation, therefore, has been extensively investigated over the years as a method for producing ohmic contacts in different grades of diamond. ${ }^{6-11}$ Among different device concepts, this processing step is beneficial for a photoconductive switch operating in a high quantum efficiency mode. ${ }^{12-15}$ Specifically attractive is the vertical device structure with opposing contacts as it provides several strategies for efficient lateral illumination and takes advantage of the large breakdown electric fields reported for diamond.

Creation of ohmic contacts requires high implantation doses that are shown to result in amorphization of the diamond lattice due to a variety of induced defects ranging from vacancies and interstitials to their numerous complexes. ${ }^{16}$ Most of the defects are eliminated using post-implantation annealing and removal of a resulting graphite layer by means of chemical etching or mechanical polishing. ${ }^{8,17}$ Some of the defects like carbon vacancies are, nevertheless, known to diffuse into the bulk of the sample during the annealing step. ${ }^{18}$ Investigating effects of such surface related residual defects on carrier recombination rates in diamond device structures is difficult due to their localized nature. Extraction of surface recombination rates in addition to bulk carrier lifetimes has been demonstrated in semi-insulating substrates using time-of-flight measurements. ${ }^{19}$ In diamond, however, the hard to implement combination of low injections and low voltages is required for reliable detection of surface recombination due to very high carrier mobilities in this material. ${ }^{20}$ Additional difficulties in data interpretation can arise due to local electric fields induced by surface trapping, as shown in the case of evaporated contacts. ${ }^{21}$

In this work, we investigate surface related defects in diamond using a pump-probe technique based on the principle of spatially resolved free carrier absorption (FCA). This all optical approach does not require the presence of an applied electric field and allows probing of spatially resolved carrier lifetimes. The latter quality in combination with high excitation conditions facilitates extraction of surface recombination rates over a broad parameter range. By comparing two samples from the same electronic grade $\mathrm{CVD}$ diamond with and without implantation 
treatment, we show that local device lifetimes are dependent on sample growth history. In the case of low bulk-like concentrations of residual nitrogen, we see same or even longer local carrier lifetimes, indicating no degradation in surface quality after implantation processing. In the case of elevated concentrations of residual nitrogen, we see short carrier lifetimes that prevent quantification of surface recombination rates.

\section{EXPERIMENT}

The two investigated CVD-grown samples were purchased from Element Six and belong to the electronic grade diamond that is defined primarily by the low $<5 \mathrm{ppb}$ content of the substitutional nitrogen impurities. ${ }^{22}$ Both samples were rectangular substrates with the approximate dimensions of $\sim 2 \times 2 \times 0.5 \mathrm{~mm}$. The studied surfaces in the specimens corresponded to the (100) crystallographic plane of diamond. In one of the samples, the two opposing larger surfaces were one by one implanted across the whole area using $\mathrm{B}^{+}$ions with four different energies of $10,35,65$, and $100 \mathrm{keV}$ to the respective implantation doses of $1.2,2,2.2$, and
$3.2 \times 10^{15} \mathrm{~cm}^{-2}$. The implantation profile simulated for the given implantation conditions is shown in Fig. 1(c). Simulations were done using the Monte-Carlo based SRIM software ${ }^{23}$ running the same calculation mode as described in Ref. 24. The generated profile indicates that the contact region extends up to $200 \mathrm{~nm}$ below the surface with the average $B$ concentration across the contact (red curve) exceeding the Mott limit. Figure 1(d) shows that a significant concentration of vacancies at $>4 \times 10^{22} \mathrm{~cm}^{-3}$ is also created in the implanted region. After implantation, sample was annealed at $1450{ }^{\circ} \mathrm{C}$ that has been shown to be a sufficient temperature for the activation of $\mathrm{B}$ dopants. ${ }^{11}$ Sample was mounted in a graphitic holder, attached to a coper wired rod, and introduced into a graphitic box evacuated to $10^{-5}$ atmospheres. Temperature ramp-up time was $10 \mathrm{~min}$, holding time at $1450{ }^{\circ} \mathrm{C}$ was $1 \mathrm{~h}$, and ramp-down time was $30 \mathrm{~min}$. After annealing, the four lateral sides of the sample were polished to optical quality. After polishing, the graphitized layers present on the implanted surfaces were removed by 5-6 min etching in a saturated solution of sulfuric acid and potassium dichromate. Finally, the absence of unintended contamination on lateral surfaces was verified by
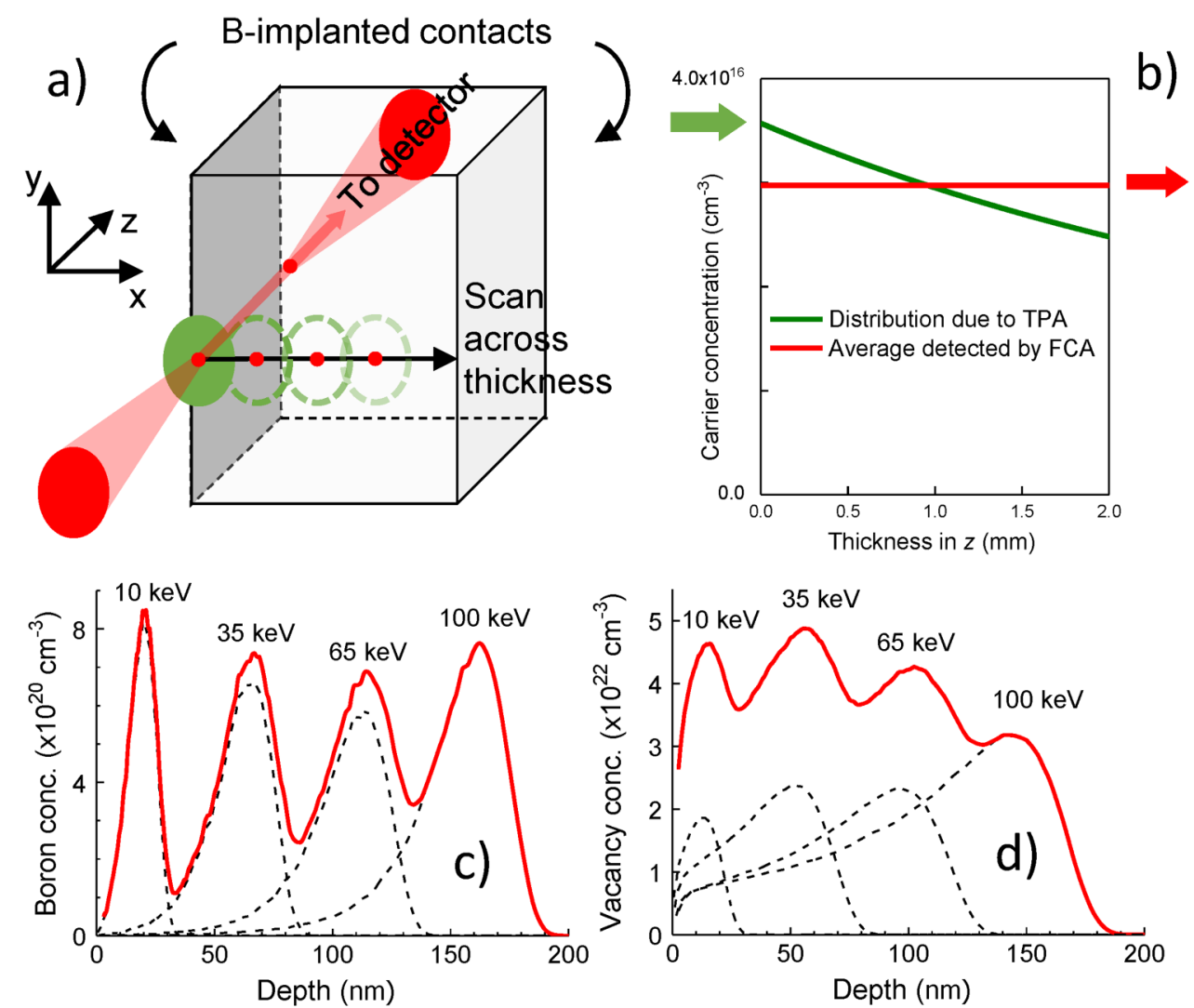

FIG. 1. (a) Schematics of the pump-probe measurement with the probe (in red) and the surrounding pump (in green) being simultaneously scanned across sample thickness in the $x$ direction to obtain spatial lifetime distributions. (b) Calculated distribution of the generated carriers (green curve) and the average concentration detected by the FCA probe (red curve) in the $z$ direction. Simulated depth profiles of implanted boron (c) and implantation produced vacancies (d) at the B-implanted contacts in the $z$ direction. In the latter graphs, individual profiles for the specific implantation energies are shown by the black dashed curves, while the overall cumulative distributions are shown by the red solid curves. 
measuring low $\sim 10^{-13} \mathrm{~A}$ dark currents between the two implanted contacts at $50 \mathrm{~V}$.

Carrier recombination measurements using the FCA pumpprobe technique have been described in detail previously. ${ }^{25}$ Experiments were performed in the collinear pump-probe geometry with the well understood resolution constrains for the effective probe volume of the Gaussian beam. ${ }^{26}$ Schematics of the measurement adopted for the diamond samples is shown in Fig. 1(a). In the picture, the B-implanted contacts correspond to the sample surfaces in the $y-z$ plane. The pump beam was oriented perpendicular to the lateral $x-y$ surface of the sample and focused to a diameter of $140 \mu \mathrm{m}$ as shown by the green circle. The excitation source was $351 \mathrm{~nm}$ laser producing $10 \mathrm{ps}$ duration pulses at $10 \mathrm{~Hz}$ repetition rate. Photon energy at this wavelength is much lower than the $5.4 \mathrm{eV}$ bandgap of diamond, and carriers are, thus, generated by the two-photon absorption (TPA) process. ${ }^{27}$ Distribution of generated carriers along the propagation path of the pump beam $(z$ direction) right after excitation was calculated using the reported TPA coefficient $\beta=0.2 \mathrm{~cm} / \mathrm{GW}$ at $3.5 \mathrm{eV}$ and the used photon flux per pulse of $50 \mathrm{~mJ} / \mathrm{cm}^{2}$. The result is shown in Fig. 1(b) by the green curve. The probe beam was aligned in the middle of the pump beam spot close to a collinear geometry and was focused to a diameter of $20 \mu \mathrm{m}$ as shown by the connected red circles. Probe source was a $50 \mathrm{~mW}$ single mode $\mathrm{cw}$-laser operating at $1.55 \mu \mathrm{m}$. The probe beam was detected by a fast InGaAs photodetector providing the average concentration of the generated carriers along the excitation path as shown by the red curve in Fig. 2(b). Intensity transients due to carrier recombination were recorded and averaged using a $2 \mathrm{GHz}$ oscilloscope. Spatial distribution of the detected signal was obtained by translating the sample in the $x$ direction perpendicular to the two implanted surfaces as shown by the arrow in Fig. 1(a).

\section{RESULTS AND ANALYSIS}

Previous reports show that carrier lifetimes in this grade of diamond are above $1 \mu \mathrm{s}$ and by orders of magnitude exceed their equivalences in lower diamond grades ranging from the polycrystalline to HTHP single crystals. ${ }^{28}$ Typical FCA transients detected in the two investigated samples are shown in Figs. 2(a) and 2(b). The color of each curve indicates a different measurement position with respect to one of the surfaces while their exact positions are indicated by the corresponding labels. The overall recombination rate is much slower for the decays detected away from the sample surfaces at 0 and $500 \mu \mathrm{m}$, but all transients show similar trends consisting of the initial fast decay and the subsequent slow decay. Carrier lifetime parameters were extracted for the two decay regions using an exponential fit as shown by the red lines. Multiple measurements were done across the sample thickness and are summarized in Figs. 3(a) and 3(b) for the two samples. The profiles reveal gradual lifetime reduction moving toward the sample surfaces. Symmetry and time evolution of the detected lifetime profiles, however, are quite different between the two samples. In the not implanted sample, the profiles are almost symmetrical and have more pronounced bowing at earlier times as shown by the fast decay component. In the implanted sample, on the other hand, the

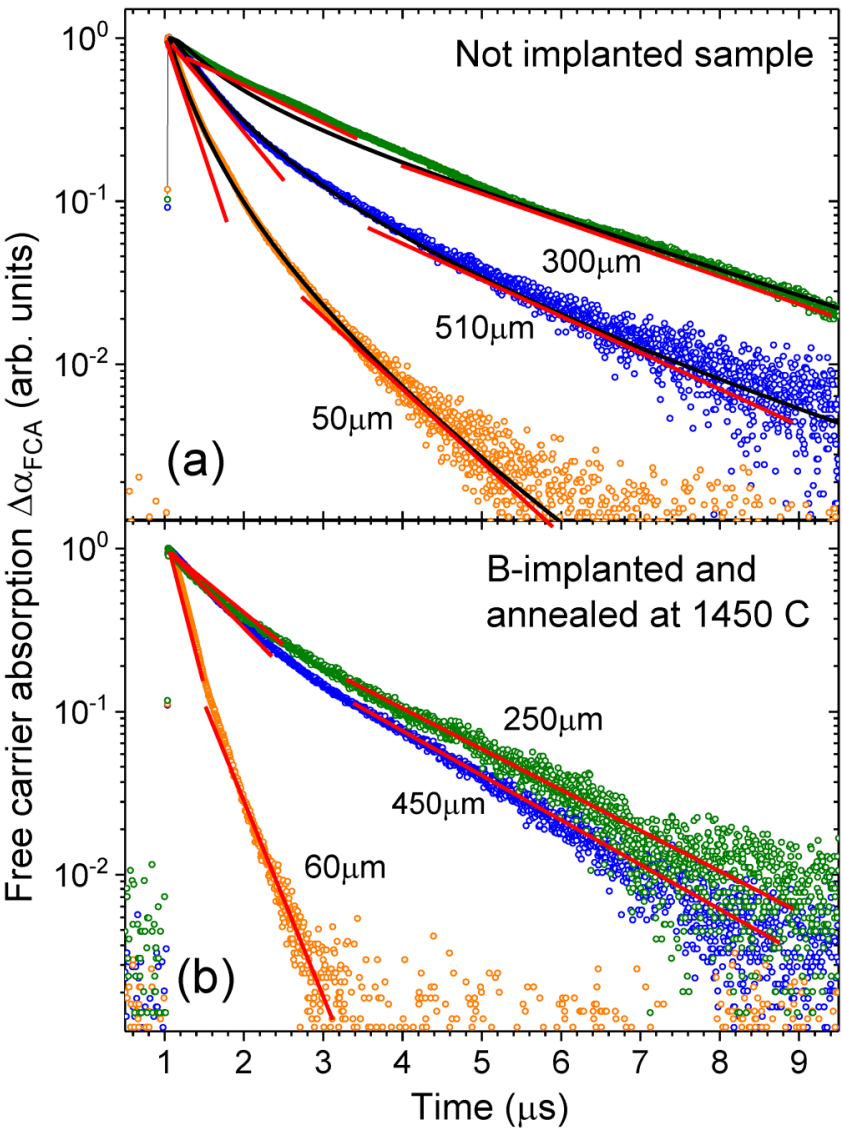

FIG. 2. FCA transients (symbols), their exponential fits (red curves), and the simulated data (black curves) at the indicated depths in (a) the not implanted and (b) implanted samples.

profiles are asymmetrical with respect to two surfaces but have similar shapes for the fast and slow decay components.

Enhanced carrier recombination rates near sample surfaces are commonly accounted for by using the surface recombination velocity, $S$, in the boundary conditions while solving the diffusion equation. If a sample volume is excited homogeneously, a simple analytical solution exists for an integrated carrier decay that approximates an initial fast carrier lifetime as $1 / \tau_{\text {Fast }}=1 / \tau_{B}+1 / \tau_{S}$ an a subsequent slow lifetime as $1 / \tau_{\text {Slow }}=1 / \tau_{B}{ }^{25,29}$ Here, $\tau_{B}$ is the bulk material lifetime and $\tau_{S}=d / S+4 d^{2} / D \pi^{2}$ is the surface lifetime where $D$ is the ambipolar diffusion coefficient and $d$ is the sample thickness along the integration direction. The guiding solid lines in Fig. 3 show, however, that in our measurements $\tau_{\text {Slow }}$ does not converge to a single parameter across the thickness for both samples. This indicates that a full numerical solution accounting for the gaussian profile of the pump beam is required to extract $S$. Such 1D calculations were carried out for each of the scan positions $x$ assuming $\tau_{\text {Slow }}(x)=\tau_{B}(x)$. A single $S$ parameter was used for the simulations near each of the surfaces and its value was adjusted to 


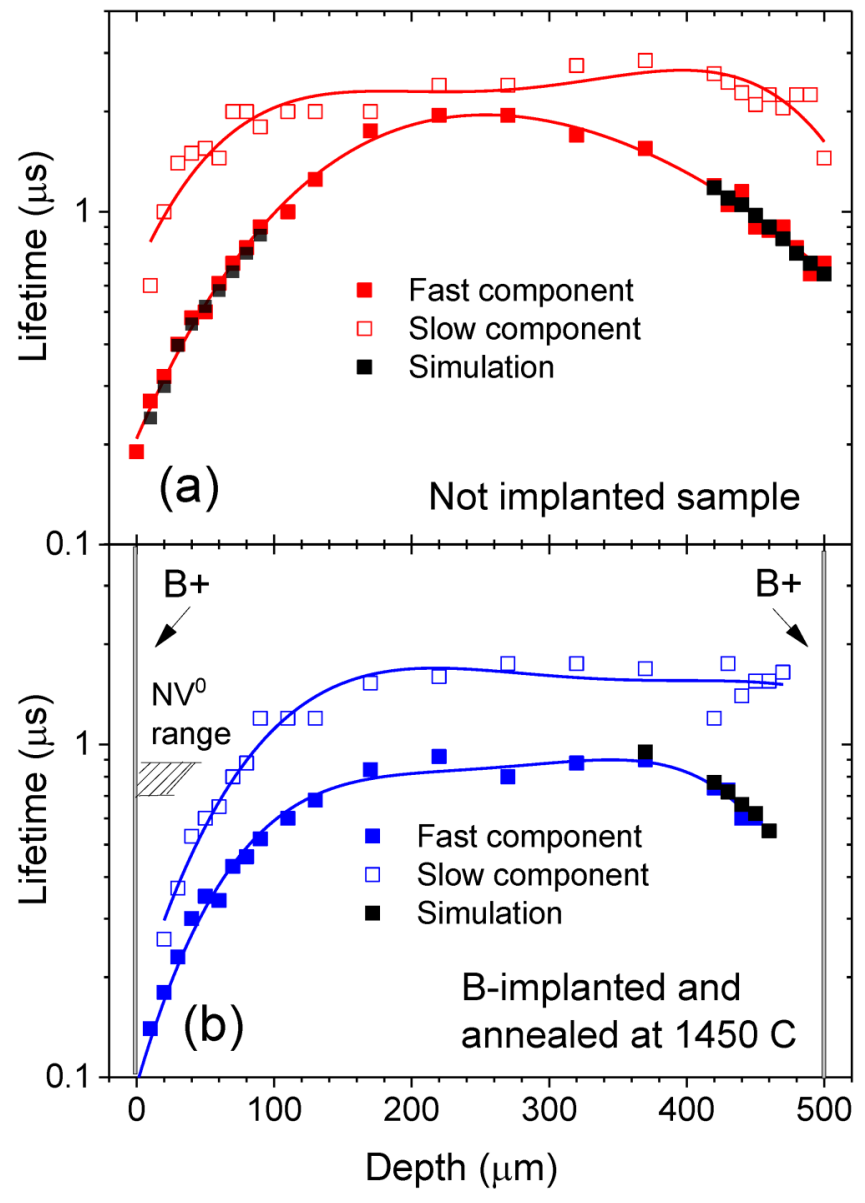

FIG. 3. Depth distributions of the extracted carrier lifetimes for the two decay components (symbols) with the polynomial guiding fits (lines) for the (a) the not implanted and (b) implanted samples.

get the best fit with the local $\tau_{\text {Slow }}$ results. In all cases, we used the ambipolar diffusion coefficient value of $D=30 \mathrm{~cm}^{2} / \mathrm{s}$ corresponding to the moderate excitation conditions. ${ }^{30}$

The simulated results are shown in Fig. 3 by the black symbols. In the not implanted sample, the observed spatial bowing of the $\tau_{\text {Fast }}$ component was reproduced by using $S_{1}=(6 \pm 2) \times 10^{3} \mathrm{~cm} / \mathrm{s}$ and $S_{2}=(3 \pm 1) \times 10^{3} \mathrm{~cm} / \mathrm{s}$ parameters for the surfaces at 0 and $500 \mu \mathrm{m}$, respectively. To the authors' knowledge, there has been no report of the $S$ parameters at a free diamond surface; however, the obtained values are well within the range of similar parameters reported for mechanically polished surfaces in other wide bandgap semiconductors. ${ }^{16,31}$ In the boron implanted sample, the results were very different for the two surfaces. For the surfaces at $500 \mu \mathrm{m}$, the $S_{2}$ parameter was in the order of $10^{2} \mathrm{~cm} / \mathrm{s}$, indicating even a slight improvement in diamond surface recombination after B-implantation and subsequent treatment. For the surface at $0 \mu \mathrm{m}$, the $S_{1}$ parameter was nondeterministic due to the contradictory requirements in data fitting.
This can be understood using the analytical expression of $\tau_{S}$ provided earlier. The large profile bowing detected at this surface would indicate the $1 / S \ll 2 d / D \pi^{2}$ condition, i.e., a carrier diffusion limited process, while the small difference between the fast and slow components would indicate the $1 / S \gg 2 d / D \pi^{2}$ condition, i.e., a surface recombination limited process. The contradiction most likely results from the breakdown of the necessary $\tau_{\text {Slow }}=\tau_{B}$ assumption in the $1 \mathrm{D}$ simulations when local carrier lifetimes become too short.

\section{DISCUSSION}

To test the hypothesis, each sample was also examined by a confocal photoluminescence (PL) setup. Samples were cooled to $77 \mathrm{~K}$ in a liquid nitrogen cryostat and excited by a $473 \mathrm{~nm} \mathrm{cw}$-laser focused to a $1 \mu \mathrm{m}$ diameter spot on a sample surface. Depth-resolved PL scans were performed in the vicinity of the pump-probe scans. It was found that in contrast to all other surfaces the surface in question shows a strong peak at $575 \mathrm{~nm}$ in the PL measurements as demonstrated by the inset in Fig. 4. The amplitude of the peak was strongest right at the surface and decreased almost exponentially away from the surface as shown in Fig. 4. Three orders of magnitude smaller PL signal was detected at the depth of $50 \mu \mathrm{m}$. The extent of this profile within the B-implanted sample is schematically illustrated in Fig. 3(b) by the dashed area.

The $575 \mathrm{~nm}$ PL spectral line in diamond is associated with the inter-center transition of the neutral nitrogen-vacancy defect $\left(\mathrm{NV}^{0}\right){ }^{32}$ The presence of this defect has been correlated with the presence of $\mathrm{N}$ which is an effective lifetime killer in diamond. ${ }^{33-35}$ We attempted a rough estimate of such $\mathrm{N}$ concentration at the surface in question using the $1 / \tau=v \sigma N_{R}$ relation, where $v$ is the thermal carrier velocity, $\sigma$ is the carrier capture cross section and

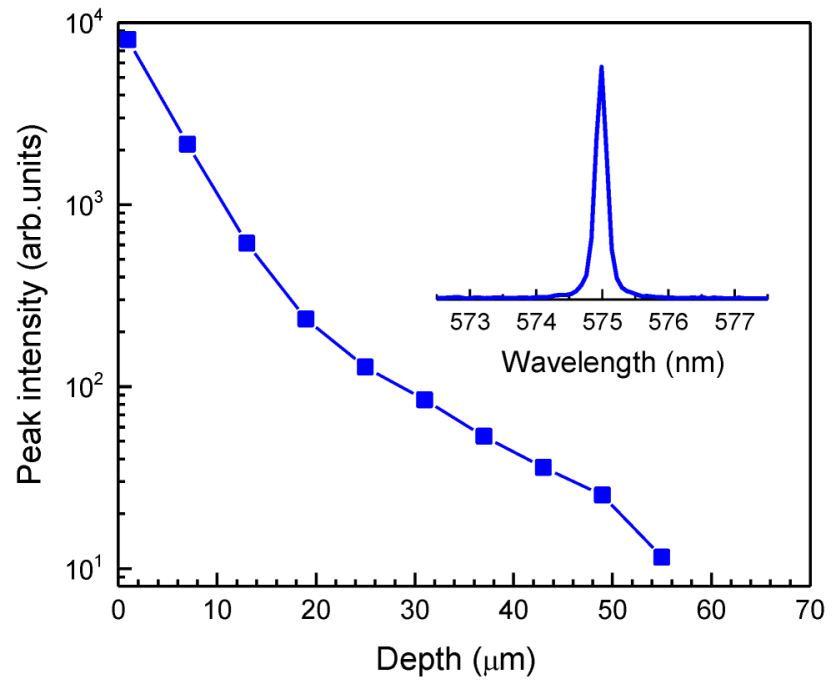

FIG. 4. Depth distribution of the PL peak intensity at $575 \mathrm{~nm}$ (inset) detected in the B-implanted sample at the $0 \mu \mathrm{m}$ surface [Fig. 3(b)]. 
$N_{R}$ is the concentration of recombination centers related to $\mathrm{N}$. Using the previously extracted $\sigma=10^{-16} \mathrm{~cm}^{2}$ for N doped CVD diamond, ${ }^{30}$ we get $N_{R}=5 \times 10^{15} \mathrm{~cm}^{3}$ from $\tau=0.2 \mu$ s extrapolated at the sample surface (Fig. 3). This value is an order of magnitude larger than $N_{R}=5 \times 10^{14} \mathrm{~cm}^{3} \quad(<3 \mathrm{ppb}$ in agreement with the vendors specification) estimated the same way from the bulk lifetime of $\tau=2 \mu \mathrm{s}$. Unfortunately, these estimates were not confirmed independently using SIMS as both values fall below the detection limit of this technique. It is likely that vacancies needed for the formation of the $\mathrm{NV}^{0}$ defect originated at the sample surface during implantation and diffused into the bulk during annealing as they have been shown to reach depths of tens of micrometers after $1 \mathrm{~h}$ at $1450^{\circ} \mathrm{C} .^{18}$ The source of $\mathrm{N}$, on the other hand, must be related to the sample itself preceding the implantation as $\mathrm{N}$ atoms have been shown to have negligible diffusion below $1700{ }^{\circ} \mathrm{C} .{ }^{36}$ In fact, unintentional $\mathrm{N}$ doping during the early $\mathrm{CVD}$ stages is well documented. ${ }^{37}$ It is not clear, however, why $\mathrm{N}$ contamination would be so localized and why elevated concentrations of $\mathrm{N}$ would be observed near one the surfaces in the investigated sample and warrant further investigations.

\section{CONCLUSION}

In summary, we detected no significant degradation of carrier lifetimes in a vertical diamond device structure exposed to highdose B-implantation and post-annealing. One of the surfaces even showed a potential improvement in surface recombination rates. The opposite surface, however, had much shorter carrier lifetimes despite being exposed to the same processing conditions. Correlation between the FCA probe and the low temperature PL scans suggests that the latter phenomenon is most likely related to the elevated concentrations of $\mathrm{N}$ at a given surface. Though the exact origin of excess $\mathrm{N}$ concentration is not clear, it was most likely introduced during growth. These findings are important for optimization of diamond photoconductive switches where very uniform carrier recombination is required across an active device structure.

\section{ACKNOWLEDGMENTS}

This work was performed under the auspices of the U.S. Department of Energy (DOE) by Lawrence Livermore National Laboratory under Contract No. DE-AC52-07NA27344 and was supported by the LLNL-LDRD Program under Project No. 17-ERD-050 (LLNL-JRNL-805200-DRAFT).

\section{DATA AVAILABILITY}

The data that support the findings of this study are available from the corresponding author upon reasonable request.

\section{REFERENCES}

1J. F. Prins, "Activation of boron-dopant atoms in ion-implanted diamonds," Phys. Rev. B 38, 5576 (1988).

${ }^{2}$ J.-P. Lagrange, A. Deneuville, and E. Gheeraert, "Activation energy in low compensated homoepitaxial boron-doped diamond films," Diamond Relat. Mater. 7, 1390 (1998).
${ }^{\mathbf{3}_{K}}$ K. Thonke, "The boron acceptor in diamond," Semicond. Sci. Technol. 18, S20 (2003).

${ }^{4}$ T. H. Borst and O. Weis, "Boron-doped homoepitaxial diamond layers: Fabrication, characterization, and electronic applications," Phys. Status Solidi A 154, 423 (1996).

${ }^{5}$ N. A. Poklonski, S. A. Vyrko, O. N. Poklonskaya, A. I. Kovalev, and A. G. Zabrodskii, "Ionization equilibrium at the transition from valence-band to acceptor-band migration of holes in boron-doped diamond," J. Appl. Phys. 119, 245701 (2016).

${ }^{6}$ G. Braunstein and R. Kalish, "Effective p-type doping of diamond by boron ion implantation,” J. Appl. Phys. 54, 2106 (1983).

7J. F. Prins, "Preparation of ohmic contacts to semiconducting diamond," J. Phys. D Appl. Phys. 22, 1562 (1989).

${ }^{8}$ V. Venkatesan, D. M. Malta, K. Das, and A. M. Belu, "Evaluation of ohmic contacts formed by B+ implantation and Ti-Au metallization on diamond," J. Appl. Phys. 74, 1179 (1993).

${ }^{9}$ R. Kalish, C. Uzan-Saguy, A. Samolloff, R. Locher, and P. Koidl, "Doping of polycrystalline diamond by boron ion implantation,” Appl. Phys. Lett. 64, 2532 (1994).

${ }^{10} \mathrm{~N}$. Tsubouchi, M. Ogura, Y. Horino, and H. Okushi, "Low-resistance p+ layer formation into diamond using heavily B ion implantation,” Appl. Phys. Lett. 89, 012101 (2006).

${ }^{11}$ J. C. Pinero, M. P. Villar, D. Araujo, J. Montserrat, B. Antunez, and P. Godignon, "Impact of thermal treatments in crystalline reconstruction and electrical properties of diamond ohmic contacts created by boron ion implantation," Phys. Status Solidi A 214, 1700230 (2017).

${ }^{12}$ P. T. Ho, C. H. Lee, J. C. Stephenson, and R. R. Cavanagh, “A diamond optoelectronic switch," Opt. Commun. 46, 202 (1983).

${ }^{13}$ S. Feng, P. T. Ho, and J. Goldhar, "Photoconductive switching in diamond under high bias field," IEEE Trans. Electron Devices 37, 2511 (1990).

${ }^{14}$ H. Yoneda, K. Ueda, Y. Aikawa, K. Baba, and N. Shohata, "Photoconductive properties of chemical vapor deposited diamond switch under high electric field strength,” Appl. Phys. Lett. 66, 460 (1995).

${ }^{15}$ E. I. Lipatov and V. F. Tarasenko, "Optoelectronic switching in diamond and optical surface breakdown,” Quantum Electron. 38, 276 (2008).

${ }^{16}$ T. Luhmann, N. Raatz, R. John, M. Lesik, J. Rodiger, M. Portail, D. Wildanger, F. Kleissler, K. Nordlund, A. Zaitsev, J.-F. Roch, A. Tallaire, J. Meijer, and S. Pezzagna, "Screening and engineering of color centers in diamond," J. Phys. D Appl. Phys. 51, 483002 (2018).

${ }^{17}$ C. Uzan-Saguy, C. Cytermann, R. Brener, V. Richter, M. Shaanan, and R. Kalish, "Damage threshold for ion-beam induced graphitization of diamond," Appl. Phys. Lett. 67, 1194 (1995).

${ }^{18}$ J. O. Orwa, K. Ganesan, J. Newnham, C. Santori, O. Barclay, K. M. C. Fu, R. G. Beausoleil, I. Aharonovich, B. A. Fairchild, P. Olivero, A. D. Greentree, and S. Prawer, "An upper limit on the lateral vacancy diffusion length in diamond," Diamond Relat. Mater. 24, 6 (2012).

${ }^{19} \mathrm{~K}$. Suzuki and H. Shiraki, "Evaluation of surface recombination velocity on CdTe radiation detectors by time-of-flight measurements," IEEE Trans. Nucl. Sci. 56, 1712 (2009).

${ }^{20}$ H. Pernegger, S. Roe, P. Weilhammer, V. Eremin, H. Frais-Kölbl, E. Griesmayer, H. Kagan, S. Schnetzer, R. Stone, W. Trischuk, D. Twitchen, and A. Whitehead, "Charge-carrier properties in synthetic single-crystal diamond measured with the transient-current technique," J. Appl. Phys. 97, 073704 (2005).

${ }^{21}$ W. Deferme, A. Mackova, K. Haenen, and M. Nesládek, "Surface states and photo-induced charge transfer on oxygen-terminated chemical vapor deposition diamond," J. Appl. Phys. 109, 063701 (2011).

${ }^{22}$ S. Mathias, Element Six Technologies, 3rd Adamas Collaboration Meeting 19 November 2014, see http://www-adamas.gsi.de/ADAMAS03/talks/Mathias_E6. pdf.

${ }^{23}$ SRIM software, see http://www.srim.org.

${ }^{24} \mathrm{~W}$. Wu and S. Fahy, "Molecular-dynamics study of single-atom radiation damage in diamond," Phys. Rev. B 49, 3030 (1994). 
${ }^{25} \mathrm{~V}$. Grivickas and J. Linnros, "Carrier lifetime: Free carrier absorption, photoconductivity and photoluminescence," in Characterization of Materials, 2nd ed., edited by E. N. Kaufmann (John Wiley \& Sons, Inc., 2012), Vol. 1, p. 658.

${ }^{26}$ J. Linnros, "Carrier lifetime measurements using free carrier absorption transients. I. Principle and injection dependence," J. Appl. Phys. 84, 275 (1998).

${ }^{27}$ J. M. P. Almeida, C. Oncebay, J. P. Siqueira, S. R. Muniz, L. De Boni, and C. R. Mendonca, "Nonlinear optical spectrum of diamond at femtosecond regime," Sci. Rep. 7, 14320 (2017).

${ }^{28}$ P. Ščajev, V. Gudelis, K. Jarašiūnas, I. Kisialiou, E. Ivakin, M. Nesládek, and K. Haenen, "Carrier recombination and diffusivity in microcrystalline CVD-grown and single-crystalline HPHT diamonds," Phys. Status Solidi A 209, 1744 (2012).

${ }^{29}$ S. S. Suvanam, K. Gulbinas, M. Usman, M. K. Linnarson, D. M. Martin, J. Linnros, V. Grivickas, and A. Hallen, "4H-SiC-Dielectric interface recombination analysis using free carrier absorption," J. Appl. Phys. 117, 105309 (2015).

${ }^{30}$ P. Ščajev, V. Gudelis, A. Tallaire, J. Barjon, and K. Jarašiūnas, "Injection and temperature dependent carrier recombination rate and diffusion length in freestanding CVD diamond," Phys. Status Solidi A 210, 2016 (2013).
${ }^{31}$ A. Galeckas, J. Linnros, M. Frischholtz, and V. Grivickas, "Optical characterization of excess carrier lifetime and surface recombination in 4H/6H-SiC," Appl. Phys. Lett. 79, 365 (2001).

${ }^{32}$ A. M. Zaitsev, Optical Properties of Diamonds: A Data Handbook (Springer Science \& Business Media, 2001).

${ }^{33}$ T. L. Nam, U. Karfunkel, R. J. Keddy, and A. G. Every, "The effects of nitrogen impurity on the radiation detection properties of synthetic diamond," Radiat. Eff. Defects Solids 116(3), 233-252 (1991).

${ }^{34}$ A. Lohstroh, P. J. Sellin, S. G. Wang, A. W. Davies, and J. M. Parkin, "Mapping of polarization and detrapping effects in synthetic single crystal chemical vapor deposited diamond by ion beam induced charge imaging," J. Appl. Phys. 101(6), 063711 (2007).

${ }^{35}$ A. Secroun, A. Tallaire, J. Achard, G. Civrac, H. Schneider, and A. Gicquel, "Photoconductive properties of lightly N-doped single crystal CVD diamond films," Diamond Relat. Mater. 16(4-7), 953-957 (2007).

${ }^{36} \mathrm{~K}$. T. Koga, J. A. Van Orman, and M. J. Walter, "Diffusive relaxation of carbon and nitrogen isotope heterogeneity in diamond a new thermochronometer," Phys. Earth Planet. Inter. 139(1-2), 35-43 (2003).

${ }^{37}$ P. M. Martineau, S. C. Lawson, A. J. Taylor, S. J. Quinn, D. J. F. Evans, and M. J. Crowder, "Identification of synthetic diamond grown using chemical vapor deposition (CVD)," Gems Gemol. 40(1), 2-25 (2004). 